\title{
Cuidados paliativos: conceptos básicos
}

\author{
Basic concepts in palliative care \\ Cuidados paliativos: conceitos básicos
}

\author{
Juan Guillermo Santacruz Escudero 1*, Luisa Fernanda Martínez Gil²
}

Recibido: 29 de septiembre de 2020. Aceptado para publicación: 15 de noviembre de 2020. https://doi.org/10.35454/rncm.v4n2.212

\section{Resumen}

Históricamente, los cuidados paliativos se han considerado como una intervención en salud, que busca mitigar el sufrimiento e integrar, de forma holística, todos los aspectos del ser: físicos, psicosociales y espirituales. Estos cuidados se aplican a los pacientes con enfermedades crónicas y amenazantes para la vida, tanto malignas como no malignas, exclusivamente en etapas finales. Esta percepción ha ido cambiando con el paso del tiempo, ya que su objetivo se centra en mejorar la calidad de vida no solo de los pacientes, sino de las familias que enfrentan este tipo de enfermedades, desde el momento del diagnóstico y a lo largo del progreso de las mismas. Los cuidados paliativos han demostrado favorecer la adecuada adherencia a los tratamientos en etapas tempranas, e incluso mejorar la sobrevida en algunas entidades, haciéndose más necesarios e intensos en estadios avanzados, a través del adecuado control sintomático y la intervención multidisciplinaria. En este artículo se presentan los conceptos básicos, un marco histórico y se abre una perspectiva para entender el papel de los cuidados paliativos en la nutrición.

Palabras clave: cuidados paliativos, calidad de vida, enfermedad crónica.

\section{Summary}

Historically, palliative care has been considered as a care intervention seeking to mitigate distress by holistically integrating all aspects of the individual: physical, psychosocial and spiritual. This care is applied to patients with both malignant and nonmalignant chronic and life-threatening diseases, exclusively in the final stages. This perception has changed over time, since currently the objective of palliative care is to improve quality of life not only of patients, but also of families who face this type of disease, from the moment of diagnosis and throughout its progression. Palliative care has been shown to favor adequate adherence to treatments in early stages, and even improve survival in some conditions, becoming increasingly necessary and intense in advanced disease stages through adequate symptomatic control and multidisciplinary intervention. This article presents the basic concepts, a historical framework, and opens a new perspective to understand the role of palliative care in nutrition.

Keywords: Palliative Care; Quality of life; Chronic disease.

\section{Resumo}

Historicamente, os cuidados paliativos têm sido considerados como uma intervenção em saúde que busca amenizar o sofrimento, integrando holisticamente todos os aspectos do ser: físicos, psicossociais e espirituais. Esses cuidados se aplicam aos pacientes com doenças crônicas e ameaçadoras para a vida, malignas e não malignas, exclusivamente em estágios finais.

Essa percepção vem mudando ao longo do tempo, pois tem como objetivo melhorar a qualidade de vida não só dos pacientes, mas também das famílias que enfrentam esse tipo de doenças, desde o momento do diagnóstico e ao longo da evolução das mesmas.

Os cuidados paliativos têm demonstrado favorecer a adesão adequada aos tratamentos em estágios iniciais e até meIhorar a sobrevida em algumas entidades, tornando-se mais necessários e intensos em estágios avançados, por meio de adequado controle sintomático e a intervenção multiprofissional.

Este artigo apresenta os conceitos básicos, um cenário histórico e abre uma perspectiva para compreender o papel dos cuidados paliativos na nutrição.

Palavras-chave: Cuidados Paliativos, Qualidade de Vida, Doença Crônica. 


\section{INTRODUCCIÓN}

La Organización Mundial de la Salud (OMS) define los cuidados paliativos como la intervención que mejora la calidad de vida de los pacientes y las familias que enfrentan los problemas asociados con las enfermedades amenazantes para la vida, a través de la prevención y alivio del sufrimiento, mediante la identificación temprana, la evaluación y el tratamiento impecable del dolor y otros problemas físicos, psicológicos y espirituales ${ }^{(1)}$. Los CP buscan atender, de forma activa y holística, a los pacientes de todas las edades con enfermedades crónicas, que generan sufrimiento debido a su severidad, y con mayor intensidad en aquellos que se encuentran cerca del final de la vida. Lo que se busca es mejorar su calidad de vida, la de sus familias y cuidadores, a través de un equipo multidisciplinario conformado por médicos, enfermeros, psicólogos, trabajadores sociales y asesores espirituales $^{(2,3)}$.

Históricamente se ha contemplado como una intervención encaminada a aliviar el sufrimiento únicamente en etapas finales de la vida; sin embargo, hoy en día, su integración temprana hace parte de las buenas prácticas en salud en la atención de todos aquellos pacientes con enfermedades crónicas complejas, oncológicas y no oncológicas, ya que ha demostrado impactar favorablemente el curso de las mismas, inclusive en términos de supervivencia, estado de ánimo y calidad de vida. Esto se logra a través del adecuado control sintomático, como lo demuestra el estudio de Temel y colaboradores en pacientes con cáncer pulmonar metastásico de células no pequeñas ${ }^{(2,4)}$.

La atención de cuidados paliativos cumple con los siguientes principios $^{(1,2)}$ :

- Proporciona alivio de síntomas de difícil control, como dolor, disnea, fatiga, náuseas, delirio y vómito persistente, distrés psicológico y espiritual, teniendo en cuenta las necesidades sociales.

- Afirma la vida y considera la muerte como parte del proceso natural.

- No intenta acelerar ni retrasar la muerte.

- Integra los aspectos espirituales y psicológicos del cuidado de la persona.

- Ofrece un sistema de soporte para ayudar a los pacientes a vivir tan activamente como sea posible hasta la muerte.

- Ayuda a las familias a adaptarse durante la enfermedad de la persona y en el duelo.

- Utiliza el trabajo en equipo para desarrollar capacidades en las personas y sus familias.
- Mejora la calidad de vida, y puede influenciar positivamente el curso de la enfermedad.

- Aplicable de forma precoz en el curso de la enfermedad, en conjunto con otros tratamientos que pueden prolongar la vida, lo que ayuda a mejorar la adherencia a los mismos.

Teniendo en cuenta lo anterior, en Colombia, la Ley 1733 de 2014 (Ley Consuelo Devis Saavedra) establece los derechos de los pacientes con enfermedades terminales, crónicas, degenerativas e irreversibles de alto impacto en la calidad de vida, así como la obligación de las entidades prestadoras de salud (EPS) e instituciones prestadoras de salud (IPS) de garantizar la atención integral para cuidados paliativos en estas circunstancias $^{(5)}$. En la Figura 1 y la Tabla 1 se muestran los dominios de atención en cuidados paliativos.

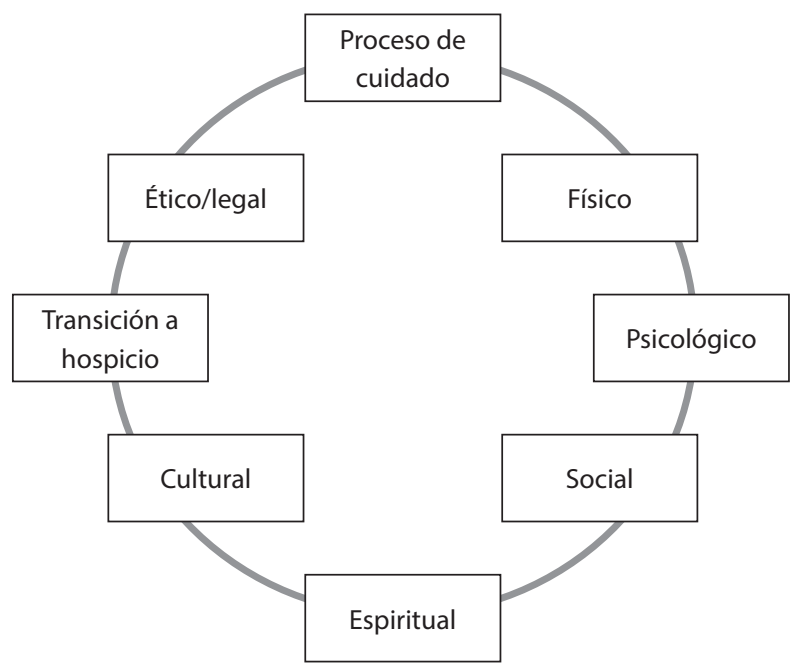

Figura 1. Dominios del cuidado paliativo. Modificado de ${ }^{(6)}$.

\section{HISTORIA}

En tiempos medievales, los hospicios (del latín Hospitium, que significa hospitalidad) se consideraban sitios de albergue para peregrinos, viajeros y extraños ${ }^{(9,10)}$. En el siglo XIX reemerge el concepto de hospicio como una institución para la atención de pacientes con enfermedades terminales bajo el cuidado, principalmente, de comunidades religiosas. Durante la época se vislumbraban los principios de los cuidados paliativos (del latín Pallium que significa capa), hallando el valor de los nuevos medicamentos analgésicos, tecnologías y los retos asociados con la atención de quienes sufrían de enfermedades avanzadas, ya que en este momento se generaba en la sociedad preocupación por el proceso de muerte ${ }^{(9,10)}$. 
Tabla 1. Áreas de desempeño de los cuidados paliativos. Adaptado de $\mathrm{de}^{(6-8)}$

Evaluación y tratamiento sintomático: dolor o síntomas físicos y psicológicos que causan estrés.

Evaluación social y espiritual: situaciones sociales o espirituales que afectan significativamente la calidad de vida.

Entendimiento de la enfermedad, pronóstico y opciones de tratamiento: apoyo al paciente, familia y cuidadores con respecto al estado actual de la enfermedad, su trayectoria y las posibilidades terapéuticas.

Identificación de los objetivos de cuidado centrados en el paciente: necesidades del paciente y su familia de acuerdo con sus valores y creencias, lo cual incluye elección del tratamiento, participación en la toma de decisiones sobre avances en maniobras o tratamientos en caso de deterioro y manifestación de voluntades anticipadas. En este sentido, la normatividad colombiana cuenta con la resolución 2665 de 2018, en la cual se establece que toda persona sana o enferma, en pleno uso de sus facultades legales mentales, puede suscribir un documento de voluntades anticipadas (DVA), el cual resguarda y garantiza el principio de autonomía en torno a la toma de decisiones sobre su salud, y que puede expresarse a través de comunicación escrita, videos o audios, o cualquier forma de comunicación, teniendo la misma validez si se suscribe ante notario, 2 testigos o ante el médico tratante.

Los avances médicos del siglo XX ignoraron el sufrimiento de los pacientes con enfermedades terminales. Es por esto por lo que entre 1950-1960 surge en Dame Cicely Saunders, enfermera, médica y trabajadora social $^{(11)}$, la inquietud del cuidado a estos pacientes, lo que cimentó las bases del movimiento moderno del cuidado paliativo y hospicio. Su abordaje del tratamiento del dolor y otros síntomas se basa en el reconocimiento de la dimensión multidimensional del sufrimiento bajo el concepto de "dolor total" y de la necesidad de integrar los aspectos físicos, emocionales y espirituales tanto del enfermo como de su familia.

El término de cuidado paliativo se utilizó, bajo el contexto actual, por primera vez por el cirujano canadiense Balfour Mount en 1975, para evitar la palabra hospicio, que en ese momento gozaba de mala reputación, por lo que significaba en tiempos antiguos. En el hospital Royal Victoria, su objetivo fue establecer un servicio de atención integral intrahospitalario, ambulatorio y domiciliario, que también apoyara la etapa de duelo, combinado con investigación y docencia, concepto que se mantiene hasta nuestros días ${ }^{(9,10)}$.

\section{MODELOS DE ATENCIÓN}

El modelo de atención paliocéntrico se enfoca en la provisión de acuerdo con la complejidad de los pacientes y el escenario de atención ${ }^{(7,13)}$ :

- Cuidados paliativos primarios: los provee todo el personal de salud a través del tratamiento sintomático inicial y de soporte. No se requiere formación específica. Se lleva a cabo en todos los niveles de atención.
- Cuidados paliativos secundarios: se lleva a cabo por equipos especializados en cuidado paliativo en pacientes con necesidades más complejas de cuidado tanto en el ámbito intrahospitalario como en consulta externa.

- Cuidados paliativos terciarios: en este nivel, el equipo de cuidados paliativos se desempeña como el servicio tratante de los pacientes con las necesidades más complejas de atención: aquellos hospitalizados en unidades de cuidados paliativos y hospicio, este último enmarcado en la filosofía de cuidado para los pacientes con enfermedades terminales oncológicas y no oncológicas con pronóstico de vida igual o menor de 6 meses.

En la Tabla 2 y la Figura 2 se muestran los componentes de los cuidados paliativos según el ámbito y la profundización en el conocimiento.

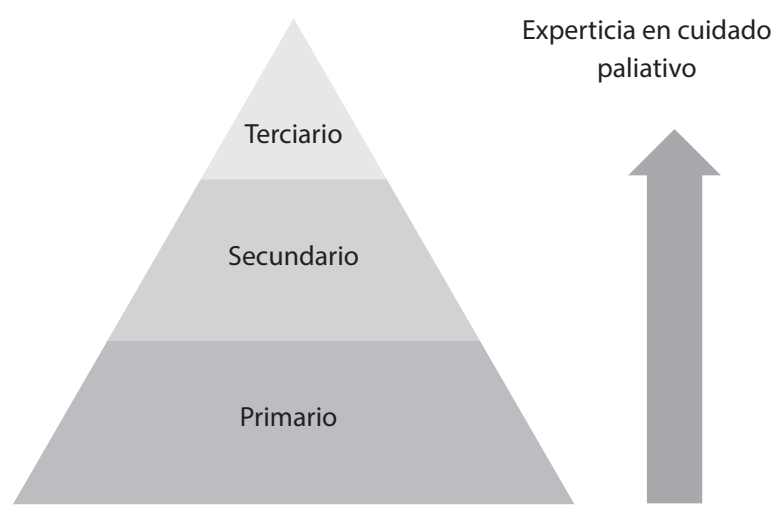

Figura 2. Componentes de los cuidados paliativos, según el ámbito y la profundización en el conocimiento. Modificada de ${ }^{(12)}$. 
Tabla 2. Habilidades en cuidados paliativos. Modificada de ${ }^{(14)}$

\begin{tabular}{|l|}
\hline Primario \\
\hline - Tratamiento básico del dolor y otros síntomas \\
- Tratamiento básico de ansiedad y depresión \\
- Discusiones básicas sobre pronóstico, objetivos de \\
tratamiento, sufrimiento, código de no reanimación \\
\hline Especializado \\
\hline - Tratamiento de dolor y síntomas refractarios \\
- Tratamiento de depresión o ansiedad complejas, duelo y \\
sufrimiento existencial \\
- Asistencia en la resolución de conflictos con respecto a los \\
objetivos o métodos de tratamiento \\
- Asistencia en casos de determinación de futilidad \\
terapéutica
\end{tabular}

\section{QUIÉNES SE BENEFICIAN DE LA ATENCIÓN POR CUIDADO PALIATIVO}

- Pacientes con enfermedades crónicas no oncológicas $^{(15,16)}$ : falla cardíaca, demencia, síndrome de fragilidad, enfermedad hepática avanzada, enfermedad pulmonar obstructiva crónica, enfermedad renal crónica terminal, enfermedades neurológicas progresivas, como esclerosis lateral amiotrófica, esclerosis múltiple, enfermedad de Parkinson y enfermedad cerebrovascular.

- Pacientes con cáncer ${ }^{(17)}$ :

- Desde el momento del diagnóstico y en plazo máximo de 8 semanas desde el momento del mismo.

- Con síntomas ya sean físicos, psicológicos o espirituales, que afectan la calidad de vida, independiente del estadio.

- Pacientes con progresión de la enfermedad con presencia de metástasis, independiente del tipo de cáncer.

- Pacientes que no se benefician de tratamiento sistémico con intención curativa.

- Pacientes con neoplasias con expectativa de vida limitada, por ejemplo, carcinoma hepatocelular.

\section{CUIDADOS PALIATIVOS EN NIÑOS}

$\mathrm{Al}$ igual que en los adultos, los cuidados paliativos buscan abordar de forma holística, y a través de un grupo interdisciplinario, a los niños y adolescentes que sufren enfermedades amenazantes para la vida, así como apoyar a sus familias y cuidadores, en la búsqueda de disminuir el sufrimiento y mejorar la calidad de vida ${ }^{(18,19)}$. Sin embargo, a diferencia de los cuidados paliativos en adultos, los profesionales y servicios especializados en niños son escasos.
La población de niños que recibe cuidados paliativos refleja los porcentajes de mortalidad infantil: $41 \%$ menores de 1 año, $42 \%$ de 2 a 17 años, y $13 \%$ mayores de 18 años. Más del $55 \%$ de los niños que recibe cuidados paliativos tiene más de una comorbilidad, que incluye enfermedades congénitas (41\%), enfermedades neuromusculares (39\%) y cáncer (20\%), incluso, en algunos casos, diagnósticos prenatales, siendo necesaria la intervención previa al nacimiento ${ }^{(18,19)}$.

En los niños, la calidad de vida se determina por la relación con sus padres y cuidadores, así como los hitos del desarrollo, lo que hace que algunos tengan una sobrevida mayor del $70 \%$ luego de 1 año de haber recibido por primera vez atención por cuidados paliativos. Esto refleja que en este grupo de pacientes se contemplan intervenciones avanzadas en relación con las vías de alimentación, e inclusive procedimientos invasivos, como traqueostomía o gastrostomía, ya que la trayectoria de la enfermedad y declive en muchos casos no es lineal ${ }^{(18,19)}$. Adicionalmente, la toma de decisiones va evolucionando a medida que el niño avanza en edad, siendo inicialmente tomadas por sus padres o cuidadores, $y$ a medida que se va teniendo consciencia de la enfermedad y la muerte se vincula al niño o adolescente.

\section{CUIDADOS PALIATIVOS Y LA NUTRICIÓN}

El incorporar los conceptos de los cuidados paliativos en todos los actores del cuidado en salud, incluidos los profesionales en nutrición, busca avanzar en los paradigmas de interacción entre los equipos, y acortar las brechas con respecto a la toma de decisiones. Esto incluye también el soporte nutricional en escenarios como el retiro o la continuidad del mismo, en pacientes con falla orgánica, o avance de enfermedades crónicas (nutrición parenteral en obstrucción intestinal maligna avanzada, pertinencia de nutrición artificial por gastrostomía en demencia terminal, entre otros). Las acciones deben realizarse validando todos los puntos de vista, y buscando facilitar la comunicación bajo la premisa del mejor beneficio para el paciente, apoyadas siempre en los principios bioéticos de respeto de la autonomía del paciente, beneficencia, no maleficencia y justicia. Estos últimos ayudan a superar los posibles dilemas y las barreras para el cambio sobre las indicaciones y el beneficio de la misma ${ }^{(20)}$.

\section{Financiamiento}

Los autores no recibieron ningún apoyo financiero para la investigación, autoría o publicación de este artículo. 


\section{Conflicto de intereses}

Los autores declaran no tener conflictos de interés en la investigación, autoría o publicación de este artículo.

\section{Declaración de autoría}

LFMG realizó la revisión, escritura, lectura y aprobación del manuscrito final. JGSE y LFMG realizaron la lectura y aprobación del manuscrito final.

\section{Referencias bibliográficas}

1. Guía de práctica clínica para la atención de pacientes en cuidado paliativo (adopción) [Internet]. Ministerio de Salud y Protección Social. 2016. (Consultado el 2 de septiembre de 2020). Disponible en: http://gpc.minsalud.gov.co/gpc sites/Repositorio/Otros_conv/GPC_paliativo/Version_ cortapaliativo2016_04_20.pdf

2. Radbruch L, De Lima L, Knaul F, Wenk R, Ali Z, Bhatnaghar $\mathrm{S}$, et al. Redefining palliative care - A new consensus-based definition. J Pain Symptom Manage. 2020;60(4):754-64. doi: https://doi.org/10.1016/j.jpainsymman.2020.04.027.

3. Swetzs KM, Kamal AH. Palliative care. Ann Intern Med. 2018;168(5):ITC33-48. doi: https://doi.org/10.7326/ AITC201803060.

4. Temel JS, Greer JA, Muzikansky A, Gallagher ER, Admane $S$, Jackson VA, et al. Early palliative care for patients with metastatic non-small-cell lung cancer. $\mathrm{N}$ Engl J Med. 2010;363(8):733-42. doi: https://doi.org/10.1056/ NEJMoa1000678.

5. Ley 1733 de 2014. Diario Oficial No. 49.268 [Internet]. Congreso de la República. Colombia; 8 de septiembre de 2014. (Consultado el 2 de septiembre de 2020). Disponible en: http://www.secretariasenado.gov.co/senado/basedoc/ ley_1733_2014.html

6. DeGroot L, Koirala B, Pavlovic N, Nelson K, Allen J, Davidson $\mathrm{P}$, et al. Outpatient palliative care in heart failure: an integrative review. J Palliat Med. 2020;23(9):1257-69. doi: 10.1089/ jpm.2020.0031.

7. Weissman DE, Meier DE. Identifying patients in need of a palliative care assessment in the hospital setting: a consensus report from the Center to Advance Palliative Care. J Palliat Med. 2011;14(1):17-23. doi: 10.1089/jpm.2010.0347.

8. Resolución 2665 de 2018 [Internet]. Ministerio de Salud y Protección Social. Colombia; 2018. (Consultado el 2 de septiembre de 2020). Disponible en: https://www.minsalud.gov.
co/Normatividad_Nuevo/Resoluci\%C3\%B3n\%20No.\%20 2665\%20de\%202018.pdf

9. Brooksbank M. Palliative care: where have we come from and where are we going? Pain. 2009;144(3):233-5. doi: 10.1016/j. pain.2009.06.010.

10. Knaul FM, Farmer PE, Krakauer EL, De Lima L, Bhadelia A, Jiang Kwete $\mathrm{X}$, et al. Alleviating the access abyss in palliative care and pain relief-An imperative of universal health coverage: the Lancet Commission report. Lancet. 2018;391(10128):1391454. doi: 10.1016/S0140-6736(17)32513-8.

11. Richmond C. Dame Cicely Saunders. BMJ. 2005;331(7510):238. doi: https://doi.org/10.1136/ bmj.331.7510.238.

12. Hui D, Bruera E. Models of integration of oncology and palliative care. Ann Palliat Med. 2015;4(3):89-98. doi: 10.3978/j. issn.2224-5820.2015.04.01.

13. Report of the National Advisory Committee on PALLIATIVE CARE [Internet]. National Advisory Committee on Palliative Care. Irlanda; 2001. (Consultado el 2 de septiembre de 2020). Disponible en: http://hdl.handle.net/10147/42522

14. Quill TE, Abernethy AP. Generalist plus specialist palliative care--creating a more sustainable Model. N Engl J Med. 2013;368(13):1173-5. doi: 10.1056/NEJMp1215620.

15. Salpeter SR, Luo EJ, Malter DS, Stuart B. Systematic review of noncancer presentations with a median survival of 6 months or less. Am J Med. 2012;125(5):512.e1-6. doi: http://dx.doi. org/10.1016/j.amjmed.2011.07.028.

16. Mounsey L, Ferres M, Eastman P. Palliative care for the patient without cancer. Aust J Gen Pract. 2018;47(11):765-9. doi: 10.31128/AJGP-07-18-4625.

17. Osman H, Shrestha S, Temin S, Ali ZV, Corvera RA, Ddungu $\mathrm{HD}$, et al. Palliative care in the global setting: ASCO resourcestratified practice guideline. J Glob Oncol. 2018;4:1-24. doi: 10.1200/JGO.18.00026.

18. Jordan M, Keefer PM, Lee YLA, Meade K, Snaman JM, Wolfe $\mathrm{J}$, et al. Top ten tips palliative care clinicians should know about caring for children. J Palliat Med. 2018;21(12):1783-9. doi: 10.1089/jpm.2018.0482.

19. Kaye EC, Rubenstein J, Levine D, Baker JN, Dabbs D, Friebert SE. Pediatric palliative care in the community. CA Cancer J Clin. 2015;65(4):316-33. doi: 10.3322/caac.21280.

20. Schwartz DB, Olfson K, Goldman B, Barrocas A, Wesley JR. Incorporating palliative care concepts into nutrition practice: across the age spectrum. Nutr Clin Pract. 2016;31(3):305-15. doi: $10.1177 / 0884533615621556$. 